Habib Ammari (Ed.)

\title{
Mathematical Modeling in Biomedical Imaging I
}

Electrical and Ultrasound Tomographies, Anomaly Detection, and Brain Imaging

望 Springer 


\section{Contents}

1 Multi-frequency Electrical Impedance Tomography and Magnetic Resonance

Electrical Impedance Tomography ........................ 1

Jin Keun Seo and Eung Je Woo

$1.1 \quad$ Introduction......................................... 1

$1.2 \quad$ Electrical Impedance Tomography $\ldots \ldots \ldots \ldots \ldots \ldots \ldots \ldots \ldots, 6$

1.2.1 Inverse Problem in RC-Circuit .................. 6

1.2.2 Governing Equation in EIT .................... 8

1.2.3 EIT System and Measured Data Set ............... 12

1.2.4 The Standard Reconstruction Method:

4-Channel EIT System.......................... 15

1.2.5 Boundary Geometry Effect and Rough Analysis of Relation Between Conductivity and Potential ................................. 21

1.2.6 Frequency-Difference EIT........................ 25

1.3 Trans-Admittance Scanner for Breast Cancer Detection...... 30

1.3.1 Review on Lesion Estimation Using EIT ............ 30

1.3.2 Trans-Admittance Scanner ...................... 38

1.4. Magnetic Resonance Electrical Impedance Tomography ..... 44

1.4.1 Fundamentals in MREIT ........................ 45

1.4.2 Mathematical Framework of MREIT .............. 55

1.4.3 Other Algorithms .............................. 64

1.4.4 Challenges in MREIT and Open Problems for Its Achievable Spatial Resolution .............. 67

References.............................................. 68

\section{Time Reversing Waves for Biomedical}

Applications ............................................. 73

Mickael Tanter and Mathias Fink

2.1 Introduction....................................... 73

2.2 Time Reversal of Acoustic Waves: Basic Principles........... 74

2.3 Time Reversal Cavities and Time Reversal Mirrors .......... 76 
2.4 Time Reversal Is a Spatial and Temporal Matched

Filter of Wave Propagation .......................... 79

2.5 Iterating the Time Reversal Process $\ldots \ldots \ldots \ldots \ldots \ldots \ldots \ldots \ldots . \ldots 2$

2.6 Applications of Time Reversal....................... 84

2.6.1 Real Time Tracking and Destruction of

Kidney Stones Using Time Reversal .............. 84

2.6.2 Time Reversal and Temporal Pulse Compression..... 87

2.6.3 Transcranial Focusing Using Time

Reversal for Brain Tumours Therapy ............. 90

References................................................ 96

3 The Method of Small-Volume Expansions

for Medical Imaging $\ldots \ldots \ldots \ldots \ldots \ldots \ldots \ldots \ldots \ldots \ldots \ldots \ldots . \ldots . \ldots . \ldots$

Habib Ammari and Hyeonbae Kang

3.1 Introduction....................................... 99

3.2 Conductivity Problem............................... 102

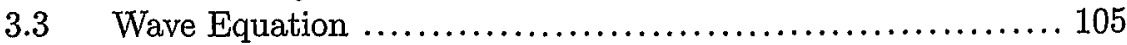

3.4 Heat Equation ....................................... 107

3.5 Modified Stokes System ............................... 109

3.6 Electrical Impedance Imaging ......................... 112

3.6.1 Detection of a Single Anomaly:

A Projection-Type Algorithm ................. 113

3.6.2 Detection of Multiple Anomalies:

A MUSIC-Type Algorithm ...................... 114

$3.7 \quad$ Impediography ........................................ 115

3.7.1 A Mathematical Model ............................ 115

3.7.2 A Substitution Algorithm ..................... 117

3.8 Magneto-Acoustic Imaging ............................ 118

3.9 Magnetic Resonance Elastography ...................... 121

3.10 Imaging by the Acoustic Radiation Force ................. 122

3.11 Infrared Thermal Imaging ............................. 124

3.11.1 Detection of a Single Anomaly ................... 125

3.11.2 Detection of Multiple Anomalies:

A MUSIC-Type Algorithm .................... 126

3.12 Bibliography and Concluding Remarks ................. 128

References.................................................... 129

4 Electric and Magnetic Activity of the Brain

in Spherical and Ellipsoidal Geometry .................... 133

George Dassios

4.1 Introduction......................................... 133

4.2 Mathematical Formulation.............................. 136

4.3 The Spherical Brain Model .............................. 141

4.4 Elements of Ellipsoidal Harmonics .......................... 149

4.5 EEG in Ellipsoidal Geometry .......................... 161 
4.6 MEG in Ellipsoidal Geometry .......................... 169

4.7 The Inverse MEG Problem .............................. 188

4.8 Open Mathematical Questions ........................... 199

References............................................... 200

5 Estimation of Velocity Fields and Propagation

on Non-Euclidian Domains: Application

to the Exploration of Cortical

Spatiotemporal Dynamics .............................. 203

Julien Lefèvre and Sylvain Baillet

5.1 Motivation: Time-Resolved Brain Imaging ............... 203

5.2 Velocity Fields and Transport on Riemannian Surfaces ...... 204

5.2.1 Vector Fields in Differential Geometry .............. 205

5.2.2 Optical Flow on a Riemannian Manifold ............ 207

5.2 .3 Regularization ................................ 207

5.2 .4 Variational Formulation ........................ 208

5.2.5 Vectorial Heat Equation ......................... 210

5.2.6 Advection on Surfaces ........................ 211

5.3 Discretization with the Finite Element Method............. 212

5.3.1 Optical Flow Equation ......................... 213

5.3.2 Pure Advection Equation ....................... 215

5.3 .3 Vectorial Diffusion........................... 216

$5.4 \quad$ Simulations......................................... 216

5.4.1 Definition of Simple Vector Fields ................ 216

5.4.2 Evaluation of the Advection Process ................ 217

5.4.3 Evaluation of the Optical Flow.................... 219

5.5 Application to Time-Resolved Brain Imaging .............. 222

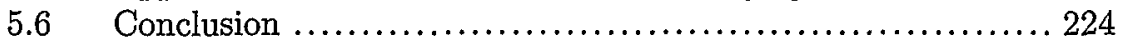

References............................................. 224

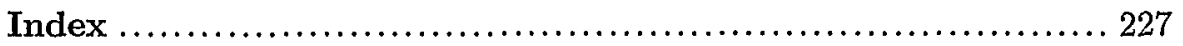

